\title{
LA COCINA TRADICIONAL. CONSIDERACIONES SOBRE SU VIGENCIA A PARTIR DEL CONTRASTE ENTRE SU PRESENCIA EN EL PASADO Y EN EL PRESENTE
}

\section{María Fernanda Padilla}

Instituto Tecnológico Particular Superior

Sudamericano

mfpadilla@sudamericano.edu.ec

(iD https://orcid.org/0000-0002-7003-7779

\section{RESUMEN}

El presente trabajo, mediante el análisis y el contraste de las relaciones que la cocina tradicional tenía con su entorno en el pasado y las que mantiene con su entorno en el presente, discierne las posturas de una cocina que se encuentra inestable por diferentes motivos. En ese sentido, este documento profundiza en la importancia de conocer nuestra tradición gastronómica para proyectarla dentro de un marco de modernidad; este trabajo se integra, así, a una tentativa más amplia que se encuentra en desarrollo, y que tiene que ver con la elaboración de una propuesta de Guía Metodológica de Procesos Creativos, en la cual consten los pasos secuenciales para que la gastronomía tradicional sea aplicada y expuesta en platos de cocina moderna. Así pues, este trabajo se concentra únicamente en el análisis y contraste de las relaciones que la cocina tradicional establece con su entorno en las dos épocas tomadas como puntos de referencia para este estudio: la primera hace referencia a las tres últimas décadas del siglo pasado y la segunda, a nuestra actualidad (2020). Para ello se fundamenta en un una metodología exploratoria y descriptiva, con un enfoque de investigación cualitativo.

Palabras Clave: c ocina tradicional, eje cultural, cocina moderna, guía metodológica
Fecha de recepción:

15 de noviembre de 2019

Fecha de aceptación:

15 de enero de 2020

\begin{abstract}
The present article, through the analysis and contrast of the relations that the Traditional Kitchen maintained with its surroundings in the past and those that it maintains with its surroundings in the present, discerns the positions of a kitchen that is wobbly for different reasons. This document deepens, thus, the importance of knowing our gastronomic tradition, to project it within a framework of modernity. Now, to develop this idea, a proposal for a Methodological Guide for Creative Processes is under development, which will include the sequential steps for traditional cuisine to be applied and exposed in modern dishes. This work, which is part of this research, focuses solely on the analysis and contrast of the relationships that traditional cuisine establishes with its surroundings in the two periods taken as a reference for the study of the subject. This is based on a qualitative research method.
\end{abstract}

Keywords: traditional cuisine, cultural axis, modern kitchen, methodological guide

Forma sugerida de citar: Padilla,M. F. (2020). La cocina tradicional. Consideraciones sobre su vigencia a partir del contraste entre su presencia en el pasado y en el presente. Convergence Tech Revista Científica. 4(1), 11-14. 


\section{INTRODUCCIÓN}

$\mathrm{S}$ i nuestra vida cotidiana se caracteriza por la modernización constante, ¿esto implica dejar de lado la cocina tradicional?, o en su defecto, ¿cómo continuar con la cocina moderna manteniendo viva la tradición? Este trabajo busca entender la relación entre las vivencias de la cocina tradicional cuencana y las tendencias propias de la gastronomía moderna.

Para intentar comprender esa relación, en este trabajo se recabó información siguiendo una metodología exploratoria y descriptiva, con un enfoque cualitativo. La información presentada, vale decir, se sustenta en referencias bibliográficas y en entrevistas estructuradas realizadas a doña Nydia Vázquez, conocedora de la cocina tradicional cuencana y al chef Diego Gutiérrez, quien se encuentra incursionando en el mundo de la cocina ecuatoriana moderna.

Se suma a estas fuentes, información proveniente de la experiencia personal y docente.

\section{ANTECEDENTES}

La definición de tradición culinaria puede llevarnos a complicadas discusiones y el objetivo de este artículo, claramente, es otro. Por ello, se optó por partir de la definición hecha por Catalina Unigarro, quien considera tradicional o típico aquello que el individuo recuerda y añora cuando, por diversos factores, no lo puede disfrutar. Se trata de una definición no muy precisa, pero que alude a lo que queda grabado en la memoria y provoca nostalgia. Se relaciona, asimismo, a lo que, por generaciones ha sido conservado, a través de su uso insistente o repetido (Unigarro, 2010, p.30).

Algo muy importante citado por la misma autora es que si se considera que la tradición es algo vivo, que evoluciona, sería valioso atender a lo que está sucediendo con las tradiciones vivas, y lo que ocurre con otras, que sólo existen como puntos de referencia en un pasado muerto. Lo tradicional, de esta manera, debe verse como un elemento que vale la pena activar mediante propuestas acordes a la modernidad.

\section{DESARROLLO}

\section{Cocina cuencana: Ayer y hoy}

Para entender más sobre la cotidianidad de la cocina cuencana se ha propuesto prestar atención a cómo se desarrollaban los principales momentos en la mesa de los cuencanos (desayuno, almuerzo, merienda) durante las últimas tres décadas del siglo $\mathrm{XX}$, y contrastarlos con el modo en que se desenvuelven hoy en día (2020). Empezamos, así, revisando el desayuno.

\section{El desayuno}

Antaño, si bien el desayuno era servido de diferentes formas, dependiendo de las costumbres de cada hogar, se pueden rescatar la abundancia de alimentos y el prolongado tiempo que se le destinaba. Así pues, algunos preferían tomar un desayuno basado en un plato fuerte que incluía estofado de carne de res o pollo y una porción de arroz, acompañado por café; un plato de mote; y, en ciertas ocasiones, de guineo (Sotomayor, 2012, p.58).

Por otro lado, estaban las familias que acostumbraban consumir un vaso de jugo de fruta de estación (generalmente de tomate de árbol), una taza de café recién pasado (obtenido del filtrado por un liencillo o chuspa sostenida por una estructura metálica) con leche, pan del día -que podía ser una crujiente palanqueta, rodilla de cristo (tugllana), mestizo, o costra- y nata.

Es más, algunos comensales utilizaban la nata como un ingrediente de la sopa magna, que se preparaba en la misma taza en la que se había consumido el café, dejando un poco de este, completándolo con pedacitos de pan, azúcar y la deliciosa nata (Cordero, 2018, p.115).

Cabe recalcar la importancia de la panadería cuencana en esta época de la historia. Para doña Nydia Vázquez, de hecho, la panadería ocupaba un espacio muy importante en la ciudad, y, dato valioso, estaban regidas casi exclusivamente por mujeres (Padilla, 2019a., párr. 3).

En la actualidad, ni el desayuno ni las otras comidas del día se desarrollan como en el pasado, sobre todo por asuntos de tiempo.

Como notorios factores del cambio tenemos a la introducción de nuevos productos alimentarios por parte del mercado (...), la creciente inserción de la mujer en el campo laboral disminuye su disponibilidad de tiempo para elaboración de alimentos (Bustamante \& Cabrera, 2014, p.14) 
De esta manera, se han reemplazado el jugo, el pan fresco y la nata por el consumo de leche o yogurt, acompañado por cereal y algo de fruta -si el tiempo lo permite-; mientras otras personas simplemente optan por servirse una taza de café instantáneo y llevar algún producto de panadería o un sándwich para comerlos en el camino.

\section{El almuerzo}

En los años ochenta, el almuerzo aún era el momento en el que la familia se reunía a compartir tanto los alimentos como las experiencias de la mañana. Era una época en la que la cotidianidad se vivía de una forma más tranquila y reposada, lo que permitía que la obtención de materia prima fuera diaria. De hecho, en algunos casos los ingredientes se conseguían de huertos propios y, en otros, provenían de mercados cercanos, lo que hacía que los materiales usados fueran frescos.

\section{El almuerzo comida estaba compuesto por.}

El primer plato: consistente en locro o sopa. En cuanto a los locros, se debe señalar como particularidad su densidad, a causa del almidón de los granos y/o la papa usados. Por su parte, la sopa, de elaboración menos densa, usualmente era elaborada a base de caldo de productos cárnicos o vegetales.

El segundo plato: generalmente constituido por una porción de carne de cerdo, res o pollo, una guarnición de arroz y una ensalada (guarnición de vegetales y/o frutas).

En relación a este último elemento (la ensalada), Daniela Sotomayor destaca que ésta variaba dependiendo del cárnico, y que en ella se utilizaba frecuentemente las alcaparras de flor de penco, los encurtidos (productos por lo general vegetales sometidos a un proceso de conservación mediante la acción de vinagre y condimentos, conservados en un envase sellado por acción del vacío), se ponían en la mesa conjuntamente con el mote" (Sotomayor, 2012, p. 62).

El postre: habitualmente constituido por almibarados caseros de frutas como el tomate de árbol, el babaco, la manzana chilena, la pera, etc. (Vintimilla, 1993, p. 371). Los almibarados eran realizados a través de una variante del método de apertización. Posteriormente, estas conservas eran almacenadas en recipientes de cristal dentro de alacenas, para ser ofrecidas al final de las comidas fa- miliares, tal y como lo recuerda Marialeja Cordero:

Alacenas, armarios y guardafrios eran espacios donde se guardaban un aserie de productos elaborados (...) en los guardafrios se ponía la leche, en los armarios: dulces del año, queso de membrillo, de guayaba, conservas apunto bien alto a que no se dañen (2018, p.63).

En la actualidad, tales actividades cotidianas han cambiado considerablemente. La adquisición de productos pre o completamente elaborados, el factor tiempo y la distancia impuestos por el trabajo a los integrantes de la familia han hecho que el desarrollo de esta comida tome otras características.

Es cada vez más común, así, que el horario laboral no permita ni elaborar los alimentos ni reunirse a consumirlos. Tal y como lo destaca el reportaje de Diana Vera, el "tiempo, la distancia y los precios son algunos de los factores que marcan los hábitos de los funcionarios en Cuenca a la hora del almuerzo" (2016, párr. 1). Han proliferado, en consecuencia, los restaurantes que ofrecen menús cíclicos, conocidos como almuerzos; estos pueden ser consumidos en el local o transportados a los domicilios a manera de viandas. Si bien estos menús están compuestos, generalmente, por entrada, plato fuerte y bebida, si se compara con el tipo de almuerzos que se preparaban antaño, se evidencia un deterioro de calidad organoléptica en cuanto a la repetición de sabores y productos. Esto último hace que los consumidores de esta clase de almuerzos, pasado un tiempo de consumo, abandonen el restaurante y realicen un verdadero peregrinaje por diversos sitios, buscando cumplir con expectativas de sabor, variedad y costo.

\section{NUEVAS PERSPECTIVAS Y CONCLUSIONES}

Antaño, la cocina tenía la importancia que merecía gracias a factores como: mayor disponibilidad de tiempo, menores distancias que cubrir al movilizarse para llegar al lugar de trabajo y fuertes núcleos familiares; todos estos factores permitían que la cocina se desarrolle de tal forma que las familias se comprometían y disfrutaban de esta actividad. Esto dio paso a que las tradiciones se mantuviesen, de generación en generación. 
En aquel entonces la cocina ecuatoriana tradicional cumplía un rol social; es decir, permitía la participación no solamente de miembros de la familia, sino también de amigos y de otro tipo de invitados. La cocina actual, sin embargo, ya no posee las mismas condiciones de aplicación que la cocina tradicional, ni respecto al proceso de preparación de alimentos ni respecto al tiempo disponible para compartir en torno a la mesa con la misma cercanía y distención.

Ahora bien, dadas estas variaciones en las condiciones actuales de vida, se torna indispensable destacar la necesidad de un conocimiento a fondo, no solo teórico sino vivencial, de las elaboraciones tradicionales base. Se destaca, en ese sentido, la postura de algunos chefs jóvenes que han sabido proyectarse hacia la cocina tradicional, para aprehender sus características esenciales y reelaborarlas en nuevas preparaciones, siempre bajo un perfil de trabajo investigativo y de respeto hacia sus particularidades culturales.

Al respecto, el chef Diego Gutiérrez opina que, bien o mal, los jóvenes cocineros están haciendo algo distinto de lo que ya se ha hecho tradicionalmente; considera, asimismo, que de la tradición se produce la vanguardia y la renovación y que, de no presentarse este proceso de innovación, habría estancamiento (Padilla, 2019b., párr.9). En esto, Gutiérrez coincide de cierta manera con el chef guayaquileño Miguel Ponce, para quien "la gastronomía ecuatoriana está en un momento innovador, en el que los cocineros jóvenes trabajan con la mentalidad de innovar y crear conceptos" (Beltrán, 2015, párr. 6).

Esto no quiere decir que abandonen la tradición gastronómica ecuatoriana. Al contrario, muchas de sus propuestas se fundamentan en un proceso de investigación variado, que no se basa sólo en la revisión bibliográfica, sino que se alimenta también de fuentes provenientes de la tradición oral (entrevistas, etc.). Es importante tomar nota de ese esfuerzo, ya que da muestras de que es sólo a través de la generación de un punto de partida sólido que se puede diseñar una propuesta asertiva y representativa de una cultura gastronómica tradicional que, pese a los cambios y renovaciones, mantenga viva la cocina tradicional.

Así pues, la cocina tradicional tiene muchas cosas que enseñar. Pero no sólo eso. Es necesario comprender su revitalización como una responsabilidad moral y profesional de los gastrónomos ecuatorianos. Sólo comprendiendo que las libertades de la creatividad no son prácticas contrarias al respeto de los sabores tradicionales, se podrá generar nuevas propuestas asertivas, que representen al mismo tiempo la riqueza de la cocina local y nacional.

\section{BIBLIOGRAFÍA}

Beltrán, J. (10 de abril de 2015). Las nuevas tendencias de la cocina ecuatoriana se muestran en un festival de Cuenca. El Comercio. Recuperado de https://www.elcomercio.com/actualidad/cuenca-azuay-gastronomia-alimentos-chefs.html

Bustamante, O, \& Cabrera, D. (2014). Cambios en los patrones alimentarios de los habitantes del barrio el vergel Cuenca-Ecuador (tesis de grado). Recuperado de http://dspace.ucuenca.edu.ec/ handle/123456789/20554

Cordero, M. (2018). Cuenca, Memoria y Cocina. Cuenca: Editorial Don Bosco-Centro Gráfico Salesiano.

Padilla, M. (2019a). [Entrevista realizada a doña Nydia Vázquez de Fernández de Córdova, autora de Ashanga]. Entrevista inédita.

(2019b). [Entrevista realizada al chef Diego Gutiérrez, propietario del restaurante La caleta]. Entrevista inédita.

Sotomayor, D. (2012). Historia de la cocina cuencana en la segunda mitad del siglo XX (Tesis de grado). Recuperado de http://dspace.ucuenca.edu.ec/bitstream/123456789/1596/1/tgas59.pdf

Unigarro, C. (2010). Patrimonio cultural alimentario. [Versión PDF]. Recuperado de https://biblio.flacsoandes.edu.ec/catalog/resGet.php?resId=52870

Vera, D. (2016, 29 junio). En Cuenca aún se conserva la tradición de reunirse en familia para almorzar. El Telégrafo. Recuperado de https://www. eltelegrafo.com.ec/noticias/septimo/1/en-cuenca-aun-se-conserva-la-tradicion-de-reunirse-en-familia-para-almorzar

Vintimilla de Crespo. (1993). Viejos secretos de la Cocina Cuencana. Cuenca: Imprenta y Offset Atlántida. 